\title{
Oriented Immobilization of Single Domain Antibodies using SpyTag/SpyCatcher Yields Improved Limits of Detection
}

George P. Anderson ${ }^{1}$, Jinny L. Liu', Lisa C. Shriver-Lake ${ }^{1}$, Dan Zabetakis ${ }^{1}$, Victor A. Sugiharto ${ }^{2,3}$, Hua-Wei Chen²,3, Cheng-Rei Lee ${ }^{2,3}$, Gabriel N. Defang², Shuenn-Jue L. Wu², Neeraja Venkateswaran', Ellen R. Goldman ${ }^{1 *}$

${ }^{1}$ Center for and Engineering, US Naval Research Laboratory, 4555 Overlook Ave SW, Washington, DC 20375, USA

${ }^{2}$ Viral and Rickettsial DiseaBiomolecular Science ses Department, Naval Medical Research Center, 503 Robert Grant Avenue, Silver Spring, MD 20910, USA

${ }^{3}$ Henry M Jackson Foundation for the Advancement of Military Medicine, 6720A Rockledge Drive, Bethesda, MD 20817, USA

${ }^{4}$ Tetracore, Inc., 9901 Belward Campus Drive Suite 300, Rockville, MD 20850, USA

* Corresponding Author: Ellen R. Goldman. Phone: 202-404-6052; email: ellen.goldman@nrl.navy.mil

Contents:

Title page

Protein sequences/BglBrick cloning scheme

Table S1. Detection of NS1 antigen in clinical samples using original assay format S3

Table S2. Detection of NS1 antigen in clinical samples using DD1-GS3K tracer

Table S3. Detection of NS1 antigen in clinical samples using DD5-GS3K tracer S5

Figure S1. Comparison of constructs and immobilization methods for SEB detection $\quad$ S6

Figure S2. LowCross Buffer (LCB) vs. PBSTB to dilute serum samples

Figure S3. Detection of the NS1 antigen from the four DENV serotypes $\quad$ S8 
Protein sequences. For the BglBrick sequences, the EF at the $\mathrm{N}$ terminus (red font) encodes an Eco RI site, The RS (green font) encodes a $\mathrm{Bg} / \mathrm{Il}$ site. Towards the $\mathrm{C}$ terminus, the GS (pink font) encodes Bam HI and the LE (blue font) encodes Xho I. All BglBricks were cloned into a pET22b expression vector modified to eliminate the single $B g /$ I/ site using the scheme shown to the right of the sequences. The DD7-DD7-ST sequence listed below was synthesized as a Nco I-Not I fragment and cloned into the standard $\mathrm{pET} 22 \mathrm{~b}$ expression vector.

>SpyCatcher BglBrick

EFARSVDTLSGLSSEQGQSGDMTIEEDSATHIKFSKRDEDGKELAGATMELRDSSGKTI

STWISDGQVKDFYLYPGKYTFVETAAPDGYEVATAITFTVNEQGQVTVNGKATKGDAHI GGGGSGGGGSGSGLEHHHHHH

>SpyTag BglBrick

EFARSAHIVMVDAYKPTKGSGLEHHHHHH

$>$ ACVE BglBrick

EFARSDVQLVESGGGLVQPGGSLRLTCAASGLIFGSYAMGWFRQAPGKAREFVAAISWS GGDTYADSVKGRFTISRDNAKNTVYLQMNSLEPEDTAVYSCAAVGSKYYISKDAKDYGY WGQGTQVTVSSAAAGGGGSGGGGSGSGLEHHHHHH
Depiction of a generic BglBrick part

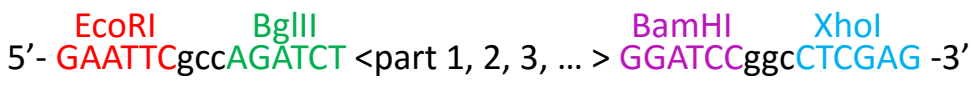

Scheme for BglBrick cloning

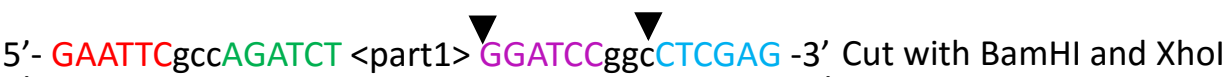
5'- GAATTCgCCAGATCT < part1> GGATCCggcCTCGAG -3', Cut with BamHI and Xho
3'- CTTAAGCggTCTAGA <part1> CCTAGGccgGAGCTC -5' Phosphatase and purify (2) 5'- GAATTCgCCA GATCT <part2> G GATCCggcCTCGAG -3' Cut with Bglll and Xhol 3'- CTTAAGCggTCTAGA <part2> CCTAGGCCgGAGCTC -5' Gel purify fragment Mix and Ligate

5'- GAATTCgccAGATCT <part1> GGATCT <part2> GGATCCggcCTCGAG -3' 3'- CTTAAGcggTCTAGA < part1> CCTAGA < part2> CCTAGGCCgGAGCTC - 5 ' 
Table S1. Detection of NS1 antigen in clinical samples using original assay format

Detection using original assay format (DD7-GS3K capture with biotinylated DD5-GS3K reporter). Bead sets 63 (negative) and 64 (DD7-GS3K) were run in duplicate. Samples positive by the sdAb assay are highlighted in yellow. Each sample was also evaluated using the InBios ELISA for NS1 detection. ELISAs were performed using $10 \mathrm{ul}$ and $1 \mathrm{ul}$ of sample. For the InBios ELISA, the positive control was 1.276, negative control was 0.115 , and cutoff control was 0.143. Samples positive by the InBios ELISA are highlighted in orange. The * indicates samples used in further testing.

\begin{tabular}{|c|c|c|c|c|c|c|c|c|c|c|}
\hline Sample ID & Sample Type & Virus Type & $\begin{array}{c}\text { Days Post- } \\
\text { onset }\end{array}$ & $\begin{array}{c}\text { PCR or IFA } \\
\text { (Acute) }\end{array}$ & $\begin{array}{c}\text { Average MFI } \\
\text { 63-Neg } \\
\text { CNTL }\end{array}$ & $\begin{array}{l}\text { Average MFI } \\
64 \text { DD7-GS3K }\end{array}$ & $\begin{array}{c}\text { Signal/ } \\
\text { Background } \\
\text { ratio* }\end{array}$ & Interpretation & $\begin{array}{l}\text { InBios NS1 OD } \\
\text { 450nm with } 10 \\
\text { ul sample }\end{array}$ & $\begin{array}{l}\text { InBios NS1 OD } \\
\text { 450nm with } 1 \text { ul } \\
\text { sample }\end{array}$ \\
\hline FSP1667 & Acute & DEN1 & 4 & IFA & 25 & 25 & 1.0 & Negative & 3.218 & 0.214 \\
\hline * FPM00604 & Acute & DEN1 & 5 & PCR & 24.5 & 33 & 1.3 & Negative & $>4$ & 3.031 \\
\hline $00244 / 14$ & Acute & DEN1 & 3 & PCR & 24 & 24.5 & 1.0 & Negative & 1.132 & 0.142 \\
\hline $00263 / 14$ & Acute & DEN1 & 4 & PCR & 26 & 28 & 1.1 & Negative & $>4$ & 0.912 \\
\hline FPI06169 & Acute & DEN2 & 4 & PCR & 24.5 & 24 & 1.0 & Negative & 0.091 & 0.077 \\
\hline FPI09737 & Acute & DEN2 & 4 & PCR & 25 & 24.5 & 1.0 & Negative & 0.096 & 0.081 \\
\hline $00976 / 13$ & Acute & DEN2 & 3 & PCR & 24 & 25 & 1.0 & Negative & 0.528 & 0.095 \\
\hline * 01259/13 & Acute & DEN2 & 5 & PCR & 24 & 24.5 & 1.0 & Negative & $>4$ & 2.853 \\
\hline * IQD3696 & Acute & DEN3 & 5 & IFA & 26.5 & 454.25 & 17.1 & Positive & $>4$ & 1.341 \\
\hline IQD4278 & Acute & DEN3 & 4 & IFA & 25 & 25.5 & 1.0 & Negative & 2.301 & 0.272 \\
\hline $00783 / 14$ & Acute & DEN3 & 4 & PCR & 27 & 524 & 19.4 & Positive & $>4$ & 1.67 \\
\hline $00242 / 15$ & Acute & DEN3 & 3 & PCR & 27.5 & 569.5 & 20.7 & Positive & $>4$ & 1.142 \\
\hline IQE7951 & Acute & DEN4 & 3 & $\mathrm{PCR} / \mathrm{IFA}$ & 24.5 & 28.5 & 1.2 & Negative & 3.882 & 0.955 \\
\hline IQA0502 & Acute & DEN4 & 4 & IFA & 24.5 & 24.5 & 1.0 & Negative & 0.095 & 0.082 \\
\hline $00283 / 15$ & Acute & DEN4 & 4 & PCR & 25.5 & 70 & 2.7 & Positive & $>4$ & 1.652 \\
\hline * 00316/15 & Acute & DEN4 & 4 & PCR & 25 & 85.5 & 3.4 & Positive & $>4$ & 2.883 \\
\hline & & Other febrile & & & & & & & & \\
\hline * 28589 & Acute & $\mathrm{Mal}+\mathrm{CHIKV}$ & & Negative & 25 & 24 & 1.0 & Negative & & \\
\hline 28787 & Acute & $\mathrm{Mal}+\mathrm{CHIKV}$ & & Negative & 24 & 24.5 & 1.0 & Negative & & \\
\hline 32178 & Acute & Mal + CHIKV_RT & & Negative & 25 & 26 & 1.0 & Negative & & \\
\hline 29577 & Acute & $\mathrm{Mal}+\mathrm{CHIKV}$ & & Negative & 25.5 & 25.5 & 1.0 & Negative & & \\
\hline$* 00139 / 15$ & Acute & $\mathrm{NHS}$ & 5 & Negative & 26 & 27.5 & 1.1 & Negative & 0.086 & 0.094 \\
\hline $00140 / 15$ & Acute & NHS & 4 & Negative & 25.75 & 25 & 1.0 & Negative & 0.077 & 0.078 \\
\hline FSS29571 & Acute & $\mathrm{NHS}$ & & Negative & 25.5 & 25 & 1.0 & Negative & & \\
\hline FSS29573 & Acute & $\mathrm{NHS}$ & & Negative & 24.5 & 25 & 1.0 & Negative & & \\
\hline
\end{tabular}


Table S2. Detection of NS1 antigen in clinical samples using DD1-GS3K tracer

Detection of NS1 antigen from DENV serotypes using both the original capture (DD7-GS3K on bead set 44) and oriented dimer capture (DD7-DD7-ST on bead sets 54 and 55). Duplicate runs were performed for each bead set and the average value reported. Positive assays (signal to background ratio of over 2) are highlighted in yellow. A series of NS1 from DENV-2 spiked into normal human serum is shown along with clinical samples. "Data type" indicates either the concentration of spiked DENV-2 or sample ID of the clinical sample. Information about the clinical samples can be found in Table S1. Each bead set was run in duplicate. In addition control bead sets were run with each sample as follows: IC: Instrument Performance; FC: Fluorescent Reporter; AC: Detection Antibody; NC: Non-specific binding

\begin{tabular}{|c|c|c|c|c|c|c|c|c|c|c|c|c|c|c|c|}
\hline DataType: & Net MFI & & & & & & & & & & & & & & \\
\hline Sample & & (44)-DD7-GS3K (Avg) & Ratio & (54)-DD7-DD7-ST (Avg) & Ratio & (55)-DD7-DD7-ST (Avg) & Ratio & (63)-IC (Avg) & Ratio & (64)-FC (Avg) & Ratio & (65)-AC (Avg) & Ratio & (66)-NC (Avg) & Ratio \\
\hline $89 \mathrm{ng} / \mathrm{ml}$ & 1780 pg & 1327.0 & 35.4 & 10085.5 & 420.2 & 9193.3 & 593.1 & 948.5 & 1.0 & 1028.0 & 1.2 & 8184.8 & 1.1 & 13.0 & 1.1 \\
\hline $44.5 \mathrm{ng} / \mathrm{ml}$ & $890 \mathrm{pg}$ & 818.0 & 21.8 & 6460.0 & 269.2 & 5912.5 & 381.5 & 951.5 & 1.0 & 918.0 & 1.0 & 7480.5 & 1.0 & 13.0 & 1.1 \\
\hline $22.25 \mathrm{ng} / \mathrm{ml}$ & $445 \mathrm{pg}$ & 438.5 & 11.7 & 3959.5 & 165.0 & 3547.3 & 228.9 & 1000.8 & 1.0 & 865.0 & 1.0 & 7640.0 & 1.0 & 11.5 & 1.0 \\
\hline $11.125 \mathrm{ng} / \mathrm{ml}$ & $222.5 \mathrm{pg}$ & 224.8 & 6.0 & 2111.5 & 88.0 & 1793.5 & 115.7 & 953.3 & 1.0 & 938.5 & 1.1 & 8124.5 & 1.1 & 11.5 & 1.0 \\
\hline $5.5625 \mathrm{ng} / \mathrm{ml}$ & $112.25 \mathrm{pg}$ & 136.8 & 3.6 & 1020.5 & 42.5 & 848.3 & 54.7 & 992.0 & 1.0 & 951.8 & 1.1 & 8013.3 & 1.1 & 11.5 & 1.0 \\
\hline $2.78125 \mathrm{ng} / \mathrm{ml}$ & $55.625 \mathrm{pg}$ & 84.3 & 2.2 & 470.0 & 19.6 & 406.3 & 26.2 & 920.5 & 1.0 & 905.5 & 1.0 & 7829.3 & 1.1 & 11.5 & 1.0 \\
\hline $1.390625 \mathrm{ng} / \mathrm{ml}$ & $27.8125 \mathrm{pg}$ & 64.5 & 1.7 & 266.5 & 11.1 & 219.0 & 14.1 & 937.8 & 1.0 & 958.0 & 1.1 & 8376.0 & 1.1 & 11.0 & 1.0 \\
\hline Normal Human Serum & $0 \mathrm{pg}$ & 37.5 & 1.0 & 24.0 & 1.0 & 15.5 & 1.0 & 960.0 & 1.0 & 889.0 & 1.0 & 7367.3 & 1.0 & 11.5 & 1.0 \\
\hline FMP00604 & & 47.5 & 1.3 & 2846.5 & 118.6 & 1924.5 & 124.2 & 943.0 & 1.0 & 1158.3 & 1.3 & 8403.5 & 1.1 & 11.0 & 1.0 \\
\hline 01259/13 & & 27.5 & 0.7 & 23.5 & 1.0 & 15.5 & 1.0 & 952.8 & 1.0 & 926.0 & 1.0 & 7551.8 & 1.0 & 11.0 & 1.0 \\
\hline IQD3696 & & 208.5 & 5.6 & 3221.3 & 134.2 & 2532.0 & 163.4 & 882.0 & 0.9 & 1085.3 & 1.2 & 8645.8 & 1.2 & 11.5 & 1.0 \\
\hline 00316/15 & & 2162.0 & 57.7 & 9107.5 & 379.5 & 8387.8 & 541.1 & 937.8 & 1.0 & 1115.0 & 1.3 & 7833.0 & 1.1 & 14.0 & 1.2 \\
\hline 28589 & & 31.5 & 0.8 & 23.3 & 1.0 & 15.0 & 1.0 & 948.5 & 1.0 & 1219.5 & 1.4 & 9301.5 & 1.3 & 10.5 & 0.9 \\
\hline 00139/15 & & 33.0 & 0.9 & 23.5 & 1.0 & 16.0 & 1.0 & 997.0 & 1.0 & 556.0 & 0.6 & 2744.5 & 0.4 & 14.0 & 1.2 \\
\hline
\end{tabular}


Table S3. Detection of NS1 antigen in clinical samples using DD5-GS3K tracer

Detection of NS1 antigen from DENV serotypes 1 and 2 using both the original capture (DD7-GS3K on bead set 44) and oriented dimer capture (DD7-DD7-ST on bead sets 54 and 55). Duplicate runs were performed for each bead set and the average value reported. Positive assays (signal to background ratio of over 2) are highlighted in yellow. A series of NS1 from DENV-2 spiked into normal human serum is shown along with clinical samples. "Data type" indicates either the concentration of spiked DENV-2 or sample ID of the clinical sample. Information about the clinical samples can be found in Table S1. Each bead set was run in duplicate. In addition control bead sets were run with each sample as follows: IC: Instrument Performance; FC: Fluorescent Reporter; AC: Detection Antibody; NC: Non-specific binding

\begin{tabular}{|c|c|c|c|c|c|c|c|c|c|c|c|c|c|c|c|}
\hline DataType: & Net MFI & & & & & & & & & & & & & & \\
\hline Sample & & (44) DD7-GS3K Avg & Ratio & (54) DD7-DD7-ST Avg & Ratio & (55) DD7-DD7-ST Avg & Ratio & (63) IC Avg & Ratio & (64) FC Avg & Ratio & (65) AC Avg & Ratio & (66) NC Avg & Ratio \\
\hline $89 \mathrm{ng} / \mathrm{ml}$ & $1780 \mathrm{pg}$ & 748.0 & 25.4 & 5744.0 & 225.3 & 4971.0 & 292.4 & 1164.5 & 1.0 & 1017.0 & 1.2 & 10998.3 & 1.1 & 14.0 & 1.2 \\
\hline $44.5 \mathrm{ng} / \mathrm{ml}$ & $890 \mathrm{pg}$ & 538.0 & 18.2 & 4358.0 & 170.9 & 3658.8 & 215.2 & 1170.0 & 1.0 & 923.5 & 1.1 & 10586.8 & 1.1 & 13.5 & 1.1 \\
\hline $22.25 \mathrm{ng} / \mathrm{ml}$ & $445 \mathrm{pg}$ & 291.0 & 9.9 & 2303.3 & 90.3 & 1914.5 & 112.6 & 1165.0 & 1.0 & 891.8 & 1.0 & 10290.3 & 1.0 & 13.3 & 1.1 \\
\hline $11.125 \mathrm{ng} / \mathrm{ml}$ & $222.5 \mathrm{pg}$ & 162.0 & 5.5 & 1248.8 & 49.0 & 1011.8 & 59.5 & 1189.0 & 1.1 & 913.5 & 1.1 & 10142.5 & 1.0 & 12.5 & 1.0 \\
\hline $5.5625 \mathrm{ng} / \mathrm{ml}$ & $112.25 \mathrm{pg}$ & 95.0 & 3.2 & 642.0 & 25.2 & 491.8 & 28.9 & 1166.0 & 1.0 & 880.5 & 1.0 & 10130.0 & 1.0 & 12.8 & 1.1 \\
\hline $2.78125 \mathrm{ng} / \mathrm{ml}$ & $55.625 \mathrm{pg}$ & 62.0 & 2.1 & 314.0 & 12.3 & 236.3 & 13.9 & 1151.8 & 1.0 & 863.3 & 1.0 & 9961.0 & 1.0 & 12.5 & 1.0 \\
\hline $1.390625 \mathrm{ng} / \mathrm{ml}$ & $27.8125 \mathrm{pg}$ & 51.3 & 1.7 & 174.0 & 6.8 & 140.0 & 8.2 & 1155.0 & 1.0 & 839.3 & 1.0 & 9866.5 & 1.0 & 12.5 & 1.0 \\
\hline Normal Human Serum & $0 \mathrm{pg}$ & 29.5 & 1.0 & 25.5 & 1.0 & 17.0 & 1.0 & 1128.5 & 1.0 & 867.3 & 1.0 & 9882.5 & 1.0 & 12.0 & 1.0 \\
\hline FMP00604 & & 35.5 & 1.2 & 1140.5 & 44.7 & 741.0 & 43.6 & 1148.5 & 1.0 & 1077.3 & 1.2 & 10231.3 & 1.0 & 13.0 & 1.1 \\
\hline $01259 / 13$ & & 28.5 & 1.0 & 25.0 & 1.0 & 16.0 & 0.9 & 1138.3 & 1.0 & 528.8 & 0.6 & 6116.0 & 0.6 & 12.0 & 1.0 \\
\hline
\end{tabular}




\section{Figure S1.}

A

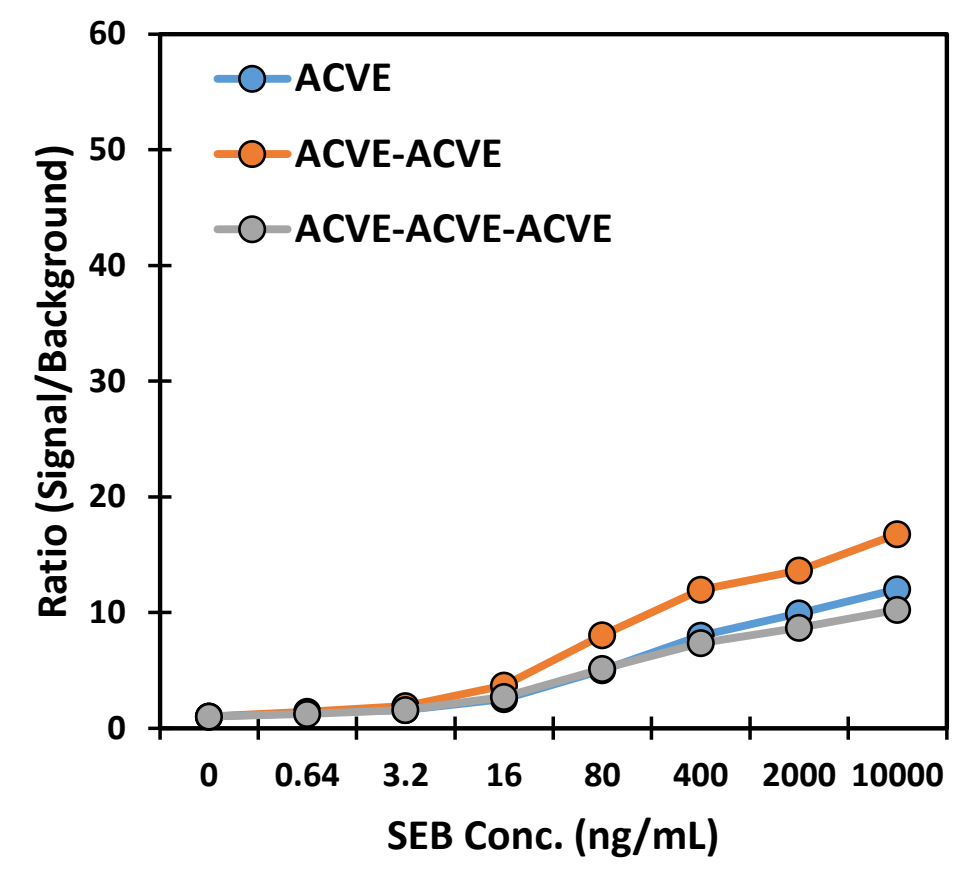

B

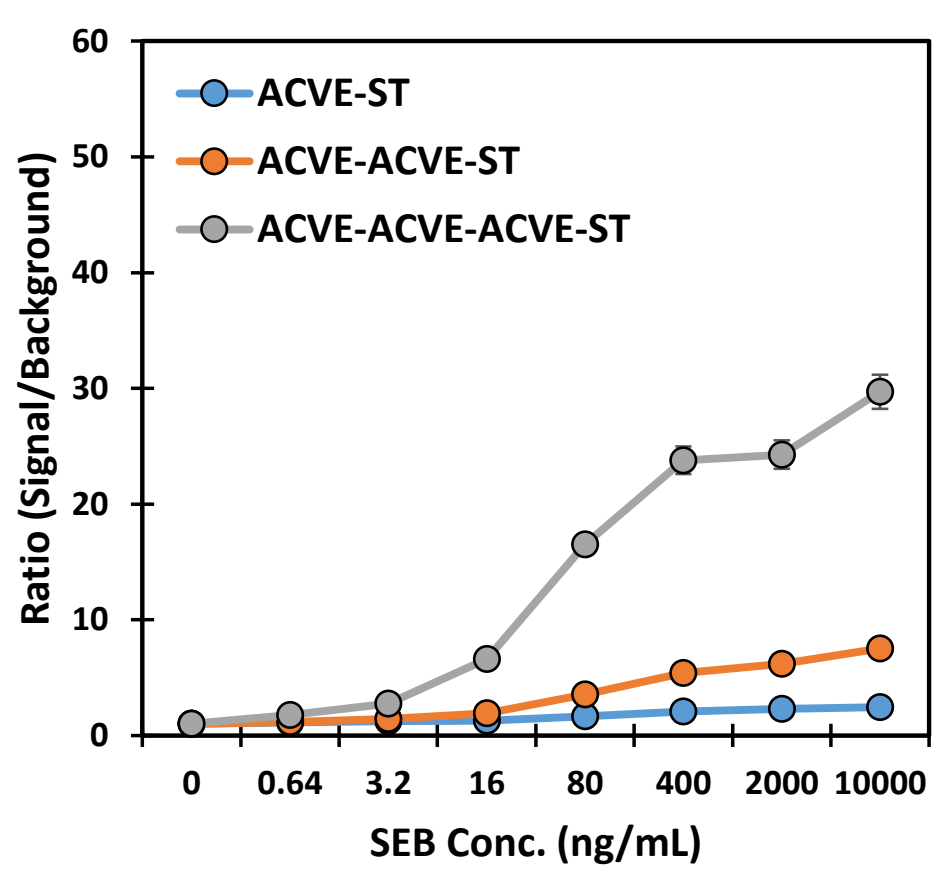

C

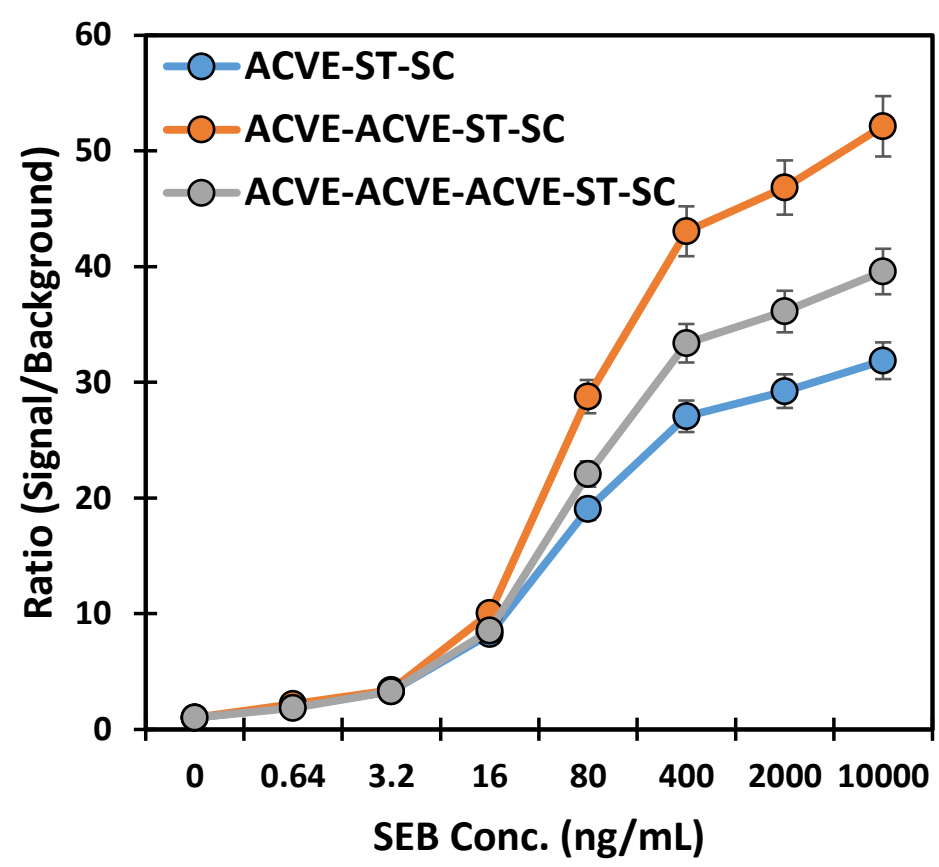

Comparison of immobilization methodology on the ratio of the signal divided by background following capture of SEB at various concentrations. (A) ACVE constructs and (B) ACVE-SpyTag (ST) constructs immobilized using conventional EDC coupling are compared to (C) ACVE-ST constructs oriented via SpyCatcher (SC) that has been covalently immobilized onto MagPlex beads. The ACVE-ACVE dimer oriented on the microsphere via a SpyTag tail fused to the $\mathrm{C}$-terminus provided the best results. Error bars represent the SEM. 
Figure S2.

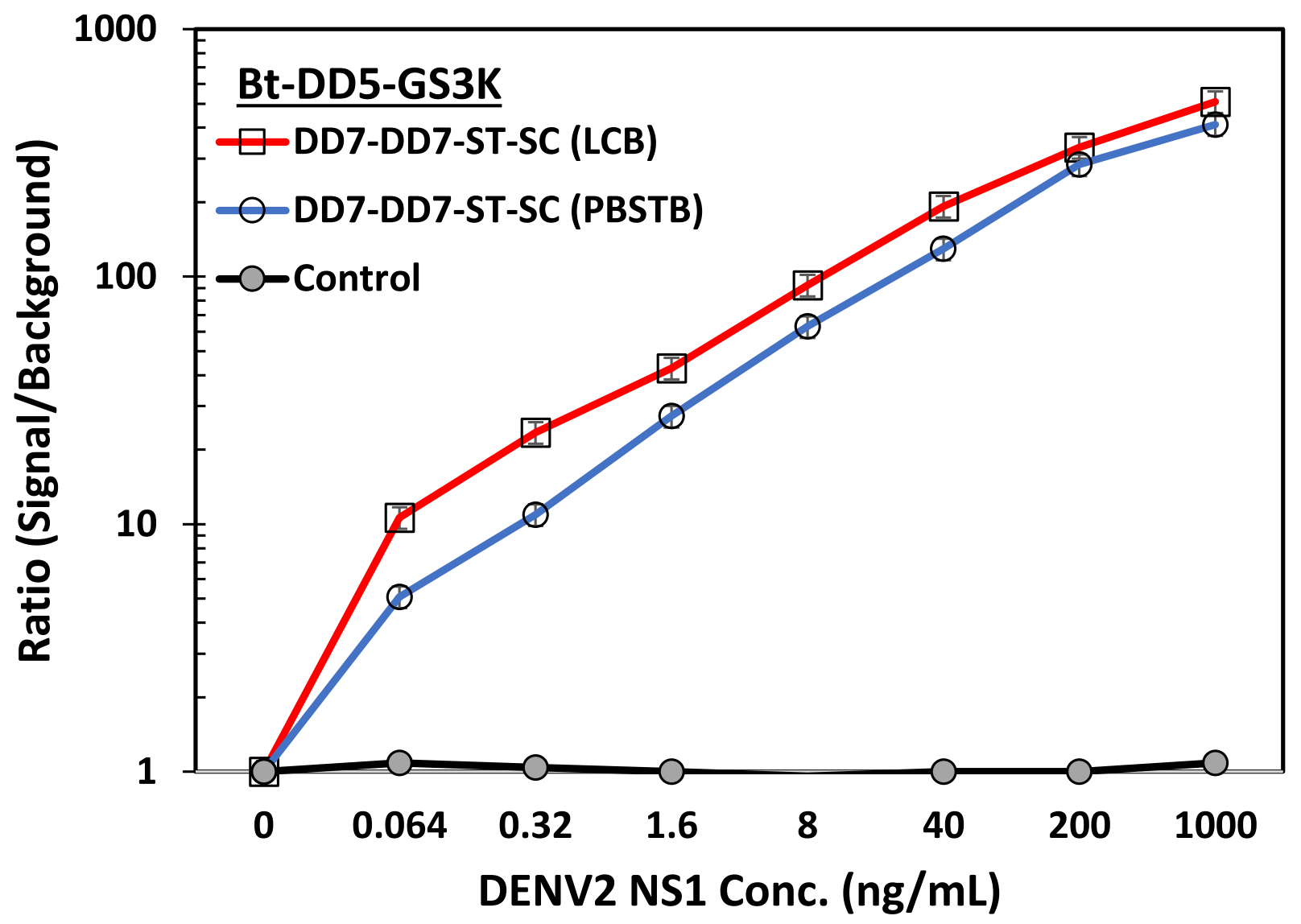

The effect of using LowCross Buffer (LCB) to dilute serum samples in comparison to PBSTB was examined. It was found that LowCross Buffer improved the signal to background by about a factor of 2 at low concentration of target. Thus all subsequent patient serum samples were diluted 1:1 with LowCross Buffer prior to testing. 


\section{Figure S3.}
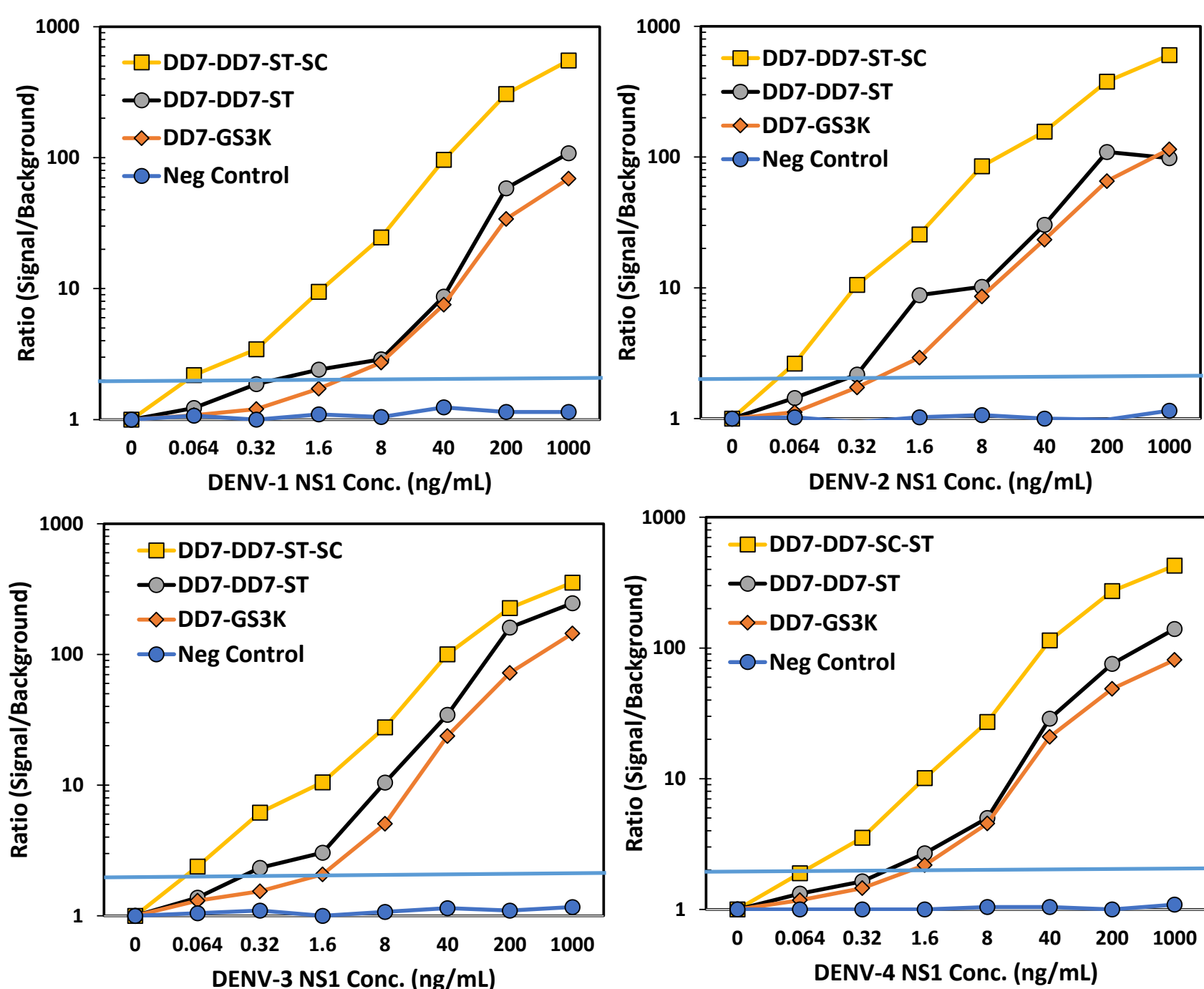

Detection of the NS1 antigen from the four DENV serotypes (DENV-1 through DENV-4). Comparison using the biotinylated DD5-GS3K reporter combined with the original DD7-GS3K capture, oriented DD7-DD7-ST-SC capture, and unoriented DD7-DD7-ST capture. In each case the DENV serotype being tested is listed on the X-axis. The blue line drawn at the signal to background ratio of 2 indicates the cutoff for labeling a sample as positive. The limit of detection of NS1 from all four DENV serotypes was $0.064 \mathrm{ng} / \mathrm{mL}$ using the oriented DD7-DDT-ST-SC capture, versus between 0.32 and $1.6 \mathrm{ng} / \mathrm{ml}$ using the unoriented DD7-DD7-ST capture, and between $1.6 \mathrm{and} 8 \mathrm{ng} / \mathrm{ml}$ using the original DD7-GS3K capture. 